\title{
Home-Based Exercise to Habitual Physical Activity Strategies for Elderly with Sarcopenia: A Literature Review
}

\author{
Ni Kadek Yuni Fridayani, ${ }^{1 *}$ Yuliana Restu Tulak, ${ }^{1}$ Cheuk Hin Ho ${ }^{2}$
}

\begin{abstract}
Background: Sarcopenia's definition are low muscle strength, low muscle quantity or quality, and low muscle performance. Several studies have found that skeletal mass can decrease as a result of a lack of physical activity and it along have to increase risk of adverse outcomes including falls, frailty, disability, morbidity and mortality. Exercise interventions is the most effective strategy for increasing the skeletal muscle mass and maintain functional independence of elderly people. In this review will discuss about how physical activity be a habitual exercise that less cost and easy to do, will be home based exercise for elderly people with sarcopenia to decrease of exacerbate.
\end{abstract}

Methods: The literature had selected studies from the following databases: PubMed, Google Scholar and PEDro. We explored evidence which relevant topic using the following database with keyword 'sarcopenia and pre-sarcopenia', 'exercise for sarcopenia and pre-sarcopenia', 'home-based exercise', 'physical activity for elderly', 'elderly with sarcopenia' and selection by inclusion and

exclusion criteria.

Results: Four studies were corresponded with home exercisebased habitual physical activity for people with pre sarcopenia and prevent sarcopenia which have been analyzed as results of the study. The habitual physical activity that can be home based exercise for prevent decreasing of muscle mass in elderly people are exercise snacking, stretching exercise, walking and tele exercise. All of the exercises showed significantly increased muscle mass and were easy to do, and those safety. The easiest is exercise snacking, but stretching exercise and walking around are more effective to increase the muscle mass and muscle strength compared to exercise snacking along.

Conclusion: Sarcopenia can be prevented in older adults with in home-based exercise from habitual physical activity such as exercise snacking, stretching exercise, walking around, and teleexercise.
'Bachelor and Professional Program of Physical Therapy, College of Medicine, Universitas Udayana, Bali, Indonesia 2University of Bradford, United Kingdom

*Corresponding author: Ni Kadek Yuni Fridayani; Bachelor and Professional Program of Physical Therapy, College of Medicine, Universitas Udayana, Bali, Indonesia; yuni.fridayani@gmail.com

Received : 2021-06-26 Accepted : 2021-10-24 Published : 2021-12-02

Keywords: elderly, habitual physical activity, home-based exercise, sarcopenia.

Cite this Article: Fridayani, N.K.Y., Tulak, Y.R., Ho, C.H. 2021. Home-Based Exercise to Habitual Physical Activity Strategies for Elderly with Sarcopenia: A Literature Review. Physical Therapy Journal of Indonesia 2(2): 73-78. D0I: 10.51559/ptji.v2i2.23

\section{BACKGROUND}

In terms of applicable criteria and cut-off values, the updated European Working Group on Sarcopenia in Older People (EWGSOP2 version 2018) definition of sarcopenia varies from the EWGSOP version 2010 definition. In its 2018 definition, EWGSOP2 uses low muscle strength as the primary parameter of sarcopenia. Muscle strength is presently the most reliable measurement of muscle function. There are three criteria to confirm the diagnosis of sarcopenia based on revision EWGSOP2 are low muscle strength, low muscle quantity or quality and low physical performance. ${ }^{1}$ The degree of sarcopenia is determined by physical performance. Physical performance is one of the most powerful indicators of older people's health outcomes. ${ }^{2}$ Sarcopenia is largely aging effect but, in many cases, other causes can be identified. Thus, category is primary and secondary which the primary sarcopenia is considered with aging, while secondary sarcopenia is causal factors other than ageing are evident. Sarcopenia can occur secondary to a systemic disease, especially one that may invoke inflammatory or any factors such as sedentary or physical inactivity. ${ }^{1}$

Sarcopenia is a major health issue among the older population, which are expected to older adults to be aware of it. Sarcopenia along have to increase risk of adverse outcomes including falls, frailty, disability, morbidity and mortality. ${ }^{3}$ One symptom causes sarcopenia is Locomotive Syndrome (LS) that high risk of developing systemic musculoskeletal disability. Major country such as Japan, China, Australia and the other countries that have based elderly population increased rapidly over recent years, that will cause nursing care cost have also increased. ${ }^{4}$ Considering all of that, sarcopenia will increase the cost of health and it will increase the need for long term as well. In addition, low muscle mass will increase risk of disability in older adults 
and elderly population. ${ }^{5}$

Epidemiology reported that sarcopenia affects about $5-13 \%$ of older adults (aged 60-70 years) and as many as $11 \%$ to $50 \%$ of those aged 80 years or older. The other observed that loses in muscle mass and function are also reported in middle-aged adults. In fact, there is a decline in muscle mass approximately $8 \%$ per decade from the age of 40 and $15 \%$ per decade from the age of $70 .{ }^{6}$ Recent systematic review and meta-analysis with data from 35 articles and 58,404 individuals around the world estimated that the overall prevalence of sarcopenia was $10 \%$ aged over $60 .{ }^{7}$

Sarcopenic individuals in nursing home reported more sedentary activities. ${ }^{6,7}$ Physical inactivity and sedentary lifestyle prevail in modern society which particularly affect the elderly population. Physical inactivity contributes to development of sarcopenia, whether disease related immobility or disability in older people. ${ }^{1}$ Only a small percentage of the elderly population participate in any leisure time physical activity. Previous reviews have shown that adult population have general opinion that exercise is time-consuming, also poor awareness of the benefits of exercise due to a lack of knowledge and low education levels. ${ }^{9}$ Although physical activity may have an indirect impact on some health aspects, it has a direct impact on muscle quality and quantity. ${ }^{10}$

Evidence suggested that exercise interventions is the most effective strategy for increasing the skeletal muscle mass and maintain functional independence in elderly people. ${ }^{11}$ The majority of studies have shown that physical activity increases muscle mass ${ }^{12}$ but the evidence about exercise as a habitual physical activity is limited. Enjoyable and convenient "exercise program" should be designed to appeal to more inactive people and encourage them to adhere to recommended exercise regimes. Home-based exercise has been recognized as a cost-effective and time-efficient alternative to center-based exercise training for maintaining muscle mass. Some studies showed that older adult population who engage in more minutes of moderate intensity or more have better physical functional performance. This physical activity can be engaging to exercise to increase mobility and maintain functional performance. a community research on 162 older adults aged 60 to 80 years old found that each additional hour of sitting increase the risk of sarcopenia up to $32 \% .{ }^{7}$ Therefore, the aim of this review is to determine what kind of physical activity be the exercise habitual that encourage at home for elderly people. The summary papers evaluating the type of home-based exercise would be beneficial in establishing methods to make it a habitual physical activity for a specific group with a sedentary lifestyle.

\section{METHODS}

This literature had selected studies from the following database; PubMed, Google Scholar and PEDro. The literatures were searched from October $1^{\text {st }} 2018$ to January $5^{\text {th }} 2019$. E-book from database were also included in the literature search. The literatures were searched with the following keywords 'sarcopenia and pre-sarcopenia', 'exercise for sarcopenia' home-based exercise', 'physical activity for elderly' and 'elderly with sarcopenia'. A hand search of the reference list of the retrieved studies from the above databases was performed for detecting manuscript. We only conducted full text studies and selected by inclusion and exclusion according the criteria below:

Inclusion criteria: 1) published in English and published in 2015-2019,2) reported about sarcopenia and pre-sarcopenia, 3) reported about exercise or home-based exercise for elderly and reference with sarcopenia and muscle mass.

Exclusion criteria: 1) result of study not reported, and 2) exercise for pre sarcopenia and sarcopenia are not reported. For the selected study conducted in flow chart below (Figure 1).

\section{RESULTS}

Fifteen studies found the eligibility criteria that are one systematic review and meta-analysis, one literature review, ten RCTs, one study protocol for RCT study, one study used cross sectional-based community (the look up 7+) and one study cross sectional study were selected. ${ }^{14-16}$ Nine studies were based on exercise for pre-sarcopenia and sarcopenia and one RCT exercise and nutritional control. One cross sectional study used community dwelling sample and other cross-sectional survey studied 6242 community-living adults to raise awareness in general population for lifestyle and risk factors of long-term conditions. ${ }^{14-17}$ All of the studies use sample are elderly population or older people and measure quality and quantity of muscle mass.

Samples of the study mostly were older people aged more than 65 years old. They were eligibility to criteria by low muscle mass, low muscle strength and low physical performance. All studies did not accept sample with any disorder like neurologic, musculoskeletal, cardiovascular, and history of surgery. The subjects were recruited using following various methods: web page and posters around campus, volunteering, and campaign.

As shown in Table 1 the intervention for home bases exercise from habitual physical activity are 


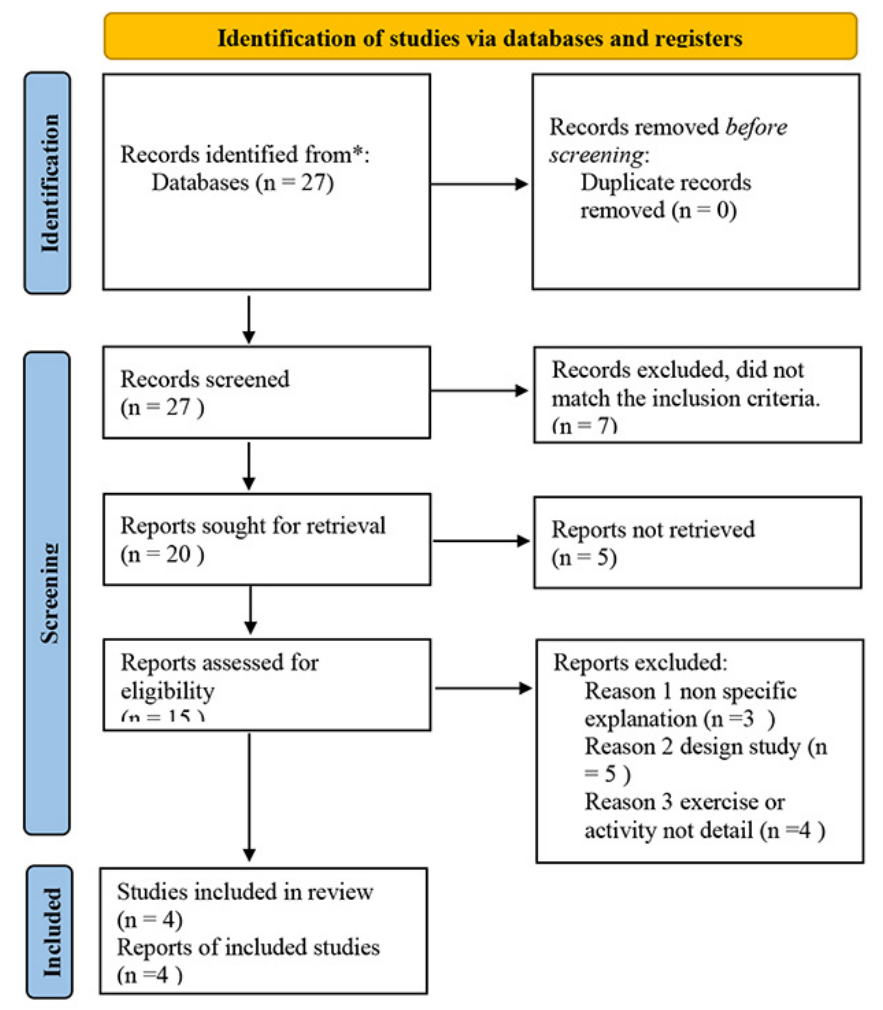

Figure 1. Flow chart diagram walking, stretching exercise, tele exercise and snacking exercise. ${ }^{14-17}$ One study had used stretching and walking exercise as part of the exercises and one study combine with nutrition control with yoghurt. One study used habitual physical activity included walking. Exercise's frequency was ranged from 28 days and 12 weeks period study.

\section{DISCUSSION}

Sarcopenia is a major component of frailty and caused by aging, disuse, altered endocrine function, chronic diseases, inflammation, insulin resistance, oxidative stress, and malnutrition, so for that we need to prevent it. ${ }^{12}$ Sarcopenia has long been associated with aging and older people, and now considered a muscle disease that has been overlooked and undertreated. ${ }^{1}$ Diagnosis sarcopenia are used mainly in research rather than in clinical practice. That was the reason EWGSOP2 stated 3 criteria (low muscle strength, low muscle quantity and low physical performance) that diagnosis is confirmed by 2 criteria met and if all met that mean sarcopenia is considered severe. ${ }^{1}$ With that new categorical and diagnosis sarcopenia is considered will easier for clinical to give education and exercise program.

Table 1. Result of Study Reviewed

\begin{tabular}{|c|c|c|c|c|}
\hline Author & Design & Dosage/frequency & Sample & Outcome \\
\hline $\begin{array}{l}\text { Hong, et } \\
\text { al., } 2016^{14}\end{array}$ & $\begin{array}{l}\text { Randomized } \\
\text { control trial }\end{array}$ & $\begin{array}{l}20-40 \text { minutes a day three } \\
\text { times per week for } 12 \\
\text { weeks }\end{array}$ & $\begin{array}{l}23 \text { elderly individuals } \\
\text { (tele-exercise group: } 11 \text {, } \\
\text { control group: } 12 \text { ), aged } \\
69 \text { to } 93 \text { years. }\end{array}$ & $\begin{array}{l}\text { - body composition using the ALST-based } \\
\text { formula } \\
\text { - functional fitness measure with The Senior } \\
\text { Fitness Test (SFT) }\end{array}$ \\
\hline $\begin{array}{l}\text { Tsekoura } \\
\text { et al., } \\
2018^{15}\end{array}$ & $\begin{array}{l}\text { Randomized } \\
\text { control trial }\end{array}$ & $\begin{array}{l}12 \text { weeks period } \\
\text { intervention with } \\
\text { Progressive group exercise } \\
\text { twice a week exercise: } \\
\text { walk } 100 \text { min per week. } \\
\text { Exercise session include: } \\
5 \text {-10 stretching, } 20-30 \\
\text { min strengthening and } 20 \\
\text { min for balance and gait } \\
\text { training }\end{array}$ & $\begin{array}{l}54 \text { elderlies ( } 47 \text { women, } \\
7 \text { men aged } 72.87 \pm 7 \\
\text { years) were randomly } \\
\text { assigned to one of } \\
\text { three interventions: } \\
\text { supervised group ( } \mathrm{n} \\
=18 \text { ), individualized } \\
\text { home-based exercise ( } \mathrm{n} \\
=18) \text { and control group } \\
(\mathrm{n}=18) \text {. }\end{array}$ & $\begin{array}{l}\text { - } \quad \text { Body composition assessment } \\
\text { - } \quad \text { Thysical function test: walking speed }(4 \mathrm{~m}) \\
\text { - } \quad \text { Quality of life assessment }\end{array}$ \\
\hline $\begin{array}{l}\text { Landi et } \\
\text { al., } 2018^{16}\end{array}$ & $\begin{array}{l}\text { Cross sectional } \\
\text { study }\end{array}$ & $\begin{array}{l}\text { Habitual physical activity: } \\
30 \text { min per session light } \\
\text { walking (leisure walking), } \\
\text { cycling, swimming running } \\
\text { and resistance training }\end{array}$ & $\begin{array}{l}6,242 \text { community-living } \\
\text { adults with the mean } \\
\text { ages } 54,4 \text { years old. }\end{array}$ & $\begin{array}{l}\text { Physical performance evaluated with part of } \\
\text { Short Physical Performance Battery (SPPB) }\end{array}$ \\
\hline $\begin{array}{l}\text { Perkin, } \\
2018^{17}\end{array}$ & $\begin{array}{l}\text { Randomized } \\
\text { control trial }\end{array}$ & $\begin{array}{l}\text { Behavioral Exercise } \\
\text { Snacking and dietary } \\
\text { supplementation yoghurt } \\
\text { for } 28 \text { days. }\end{array}$ & $\begin{array}{l}20 \text { participants } 65- \\
80 \text { years old with } \\
\text { experimental exercise } \\
\text { snacking group and } \\
\text { yoghurt only group }\end{array}$ & $\begin{array}{l}\text { - } \quad 60 \text { second sit to stand test } \\
\text { - } \quad \text { Lower limb muscle power use leg pressing } \\
\text { Lower limb movement outcome variability use } \\
\text { leg press } \\
\text { - } \quad \text { Lower limb muscle cross sectional area uses } \\
\text { peripheral quantitative computed tomography } \\
\text { - } \quad \text { oower limb muscle cross sectional tissue } \\
\text { density uses CT } \\
\text { - } \quad \text { Body composition use Dual energy X-ray }\end{array}$ \\
\hline
\end{tabular}


Exercise is needed for preventing the development of sarcopenia from pre-sarcopenia. The finding of the effectiveness of exercise in preventing sarcopenia and pre-sarcopenia in the present review confirmed its efficacy against sarcopenia, as described previously. Mechanistically, exercise is known to counter sarcopenia by reducing levels of inflammatory cytokines that induce muscle damage as well as stimulation of the rates of protein synthesis and suppression of the breakdown in muscle cells. ${ }^{18}$ However, many people nowadays find exercise boring and take time. Therefore, incorporating physical activity in home-based habitual exercise should be considered and it was the aim of this study.

The result of study showed significant decreased sarcopenia in men samples after control age, BMI, calorie intake, smoking habits, alcohol, do physical activity, and leisure time. ${ }^{19}$ Insufficient leisure time physical activity associated with sarcopenia like sports, supervised training gyms, anaerobic exercise more effectively prevent and treat people with pre sarcopenia. ${ }^{19}$ sedentary activities could result in loss of skeletal muscle and previous study showed that sedentary activities or lack of physical activity will increase the occurrence of sarcopenia. Physical activity can minimize the effects of muscle apoptosis (reduce number and size of muscle fibers) and help musculoskeletal function and increase the Qualityof-life of elderly people. ${ }^{19}$ In older years, an annual $0.5-1 \%$ muscle loss is more often which is caused by a reduction of physical activities. Physical activity has been demonstrated to be crucial for muscle mass and function maintenance. ${ }^{17}$ In New WHO guidelines (2020) recommendations for adults (1864 years) do physical activity at least average weekly volumes of 150-300 min of moderate intensity or 75-150 min of vigorous intensity. In older adults, physical activity will helps prevent falls and fallsrelated injuries, recommendation for the physical activity for older adults (aged 65 years and older) should do varied multicomponent physical activity that emphasis functional balance and strength at moderate or greater intensity on 3 or more days a week. $^{20}$

For 6-12 months of intervention, systematic evaluations of exercise programs for increasing physical activity levels in middle-aged to older adults found moderate results. Study protocol for randomized controlled trial gave the group with habitual physical activity do whatever they do every day and can participated in social or culture activity. $^{21}$ In countries with different social and cultural activities like Indonesia, elderly people are more active when engaging in social and cultural activities as they found them more enjoyable and interactive. When the elderly has some communication or have been joined the activities, that was good for their mental health and help to decrease stress. But, nowadays the elderly people have much a leisure time and do not some activity. The elderly with limited physical activity can make home based habitual physical activity form do often and participated social activities. ${ }^{21}$

Therefore, the physical activity at home that can be their habitual is the answer of that. One of the easiest and low-cost physical activity that can be habitual exercise such as walking. ${ }^{19}$ A public health research said that walking was the exercise of choice in elderly people because it was easy, low cost and could be performed in residential sites. Walking, both as an exercise and as a form of transportation. In the study, the elderly Americans said "Main thing, you don't get lazy and you don't give up" and reported that exercise must become habit forming and self-sustaining. The Chinese people in the study said that the importance of daily activities was to have routine of stretching, arm swinging and walking in early morning. However, there are the barriers to walking for elderly people such as poor environment and facilitates for walking was not good. Physical activity like walking can help digestion, blood circulation, relaxation, maintaining friendships, preventing sickness, improving life satisfaction and health. However, there are some factors that could influence the decision such as disease, enjoyment, self-efficacy, motivation, safety, fear of falling, social and family support, and also environmental factor. ${ }^{22}$

Home exercise is beneficial in improving strength in elderly individuals by using a daily program. The exercises can be completed by 5-6 repetitions, 1 minute per repetition. Recent research showed that a 6-minute home-exercise combined with walking and lower limb resistance training improved physical function in communitydwelling adults aged 60 or over with pre-sarcopenia and sarcopenia. ${ }^{18}$ One study review stated that antisarcopenia interventions could be employed by five mixed exercises including Aerobic, resistance, flexibility and balance training and combined with supplementation including: amino acid, protein and vitamin $D^{23}$ In addition, for prevent the exacerbation of decreased muscle mass, walking is the easiest and evidence showed that have the biggest impact to older people performance. ${ }^{17}$

This may present a strategy to improve recovery from reduced activity as performance tasks of daily living and should be habitual activity and the people do exercise indirectly. The Exercise Snacking is a type exercise that can do at home as home-based exercise and this exercise compose 
by 5 simple movement. ${ }^{17}$ Exercise snacking can be performed in a stable kitchen chair or usual chair in the home. This exercise program consists of 5 exercises: standing calf raises, marching on the spot, standing alternating knee bends holding onto a chair, seated alternating knee extension, and sit-to stand from a chair. This exercise gave effect if did it three times daily after patient have a meal. The exercise program is always started from sit to stand exercise. ${ }^{17}$ Exercise snacking is safe for all people and also does not require a warm up before sitting or any sport clothing or equipment apart from a chair. This exercise can also the physical activity by increasing the activity of muscle. ${ }^{18}$ If the muscle activated by the movement constantly and the movement from this exercise also safe for older people cause used chair as object and it can to prevent fall. Furthermore, this program is not time consuming, so this exercise is one choice for adult and older adult that are busy and do not have many times to do exercise. For older people, this exercise also had good benefit for increased the activity the muscle and balance.

Exergame gadgets and operating software have developed at a breakneck pace in recent years, such as the Wii Fit by Nintendo and Xbox 360 Kinect by Microsoft, which enable real time exercise feedback based on human-computer interaction technology. ${ }^{14}$ That are arrange and make the elderly fun with exercise. Study from Hong et al., 2016 showed short-term changes in sarcopenia-related factors in the elderly over the course of tele-exercise based on video conferencing. the elderly did exercise at home and follow the motion that are given by Physical Therapist (PT). ${ }^{14}$ The exercise are seem fun cause the elderly can communicate with PT and the movement that are given also simple and make the elderly not exhausted. This method exercise showed significant data for improved flexibility the elderly by repeated muscle contraction in the lower limbs as well as regular stretching. The movement in the Tele exercise majority in stretching exercise and showed good effect in increased balance and increased stimulation of myoneurons. ${ }^{14}$ But the limitation of this exercise is in country with elderly people difficult use technology and they haven't it so the exercise with tele program hard to they do. Actually, the tele program would be as both physical activity and home exercise. The older people and older adult community will have fun with the tele program as they do physical activity. But, the limitation of tele exercise is lack of technology in some country and knowledge to that. This exercise will be habit for the older people in some country that friendly with technology and the benefit the program setting indoor which is safe environment.
There are many of physical activity that the older adults and older population could do and that have a good benefit for the health especially for the skeletal muscle and to decreased a risk of fall, disability, and mortality. But just a few that will continue as a habit and exercise. As well as the result of this study showed that some physical activity considered design as home-based exercise there are walking, stretching and exercise snacking and tele exercise. All of that easy and low-cost performance. The limitation of the study was not documentation from all RCT studies for better result of study and have been applicated to population. In the future, this design of the home-based exercise from habitual physical activity can apply to population with dosage and frequency along with WHO recommendation.

\section{CONCLUSION}

The current studies have shown that sarcopenia can worsening with aging and pre-sarcopenia can affect less active elderly individuals. In order to improve people's participation and engagement, exercise strategies that can be home based habitual physical activities including walking, stretching exercise, tele exercise and exercise snacking. Exercise snacking is the easiest to be performed but walking and stretching significantly increase muscle strength compared to exercise snacking. Tele-exercise is also easy to be performed but elderly people in certain countries may find it difficult to be habitual due to unfamiliarity with new technologies.

\section{FUNDING}

This study was not funded by any grant source.

\section{CONFLICT OF INTEREST}

The author states there is no potential conflict of interest in connection with the research, authorship and or publication of this article.

\section{REFERENCES:}

1. Alfonso J. Cruz-Jentoft, Gülistan Bahat, Jürgen Bauer, Yves Boirie, Olivier Bruyère, Tommy Cederholm, Cyrus Cooper, Francesco Landi, Yves Rolland, Avan Aihie Sayer, Stéphane M. Schneider, Cornel C. Sieber, Eva Topinkova, Maurits Vandewoude, Marjolein Visser, and Mauro Zamboni. Sarcopenia: revised European consensus on definition and diagnosis. Age and Aging 2019;48:16-31

2. Jeanine M. Van A., Julian A., Carel GM. Meskers, Barbara R.N, Charlotte S., and Andra B M. Impact of using the updated EWGSOP2 definition in diagnosing sarcopenia: A clinical perspective. Archives of Gerontology and Geriatrics 90 2020: 104125

3. Tu, Di-Ya, Fa-Min Kao, Shih Tzer Tsai and Tao- Hsin Tung. Sarcopenia among the Elderly Population : A Systematic 
Review and Meta-Analysis of Randomized Controlled Trials. Healthcare 2021;9:650

4. Yamada, Tomohiro, Yu Yamato, Tomohiko H, Go Yoshida, Tatsuya Y, Tomohiro Banno, Hideyuki A, Shin Oe, Hiroki U, Koichiro Ide, Yuh W and Yukihiro Matsuyama. Impact of Habitual Exercise on Locomotive Function of Middleaged and Elderly Volunteers : A Longitudinal Study. Progress in Rehabilitation Medicine 2021 Vol 6, 20210006

5. Ancum, Jeanine M. Van, Julian A, Carel GMM, Barbara RN, Charlotte S, and Andrea BM. Impact of using the updated EWGSOP2 definition in diagnosing sarcopenia: a clinical perspective. Archives of Gerontology and Geriatrics 90 (202): 104125

6. Pardo, Pablo J M, Noelia G-G, Abraham L-V, Alejandro Espeso-G, Luis Manuel M-A, Gemma M G-G, Fransisco Javier O-C, Ana C-B, Jose Daniel J-G, Daniel V-D, Cristina C-S, Emanuele Isidori, Chiara Fossati, Fabio P, Lorenzo R, Catherine N, Audrey T, Ilvis A, Agita K-S, Juris P, Heikki H, Niko N, Leo Hokka, David J-P, and Raquel V-C. Sarcopenia, Diet, Physical Activity and Obesity in European MiddleAged and Older Adults : The LifeAge Study. Nutrients, 2021;13:8

7. Papadopoulou, Sousana K. Sarcopenia : A Contemporary Health Problem among Older Adult Populations. Nutrients 2020;12:1293

8. RN, Lee Szu-Ying, Heng Hsing Tung RN FNP, Chieh-Yu Liu, Liang-Kung Chen. Physical Activity and Sarcopenia in the Geriatric Population : A Systematic Review. Journal of the American Medical Directors Association $2018 ; 19(5)$ : 378-383

9. Evans WJ. Skeletal muscle loss: cachexia, sarcopenia, and inactivity. Am J Clin Nutr. 2010;91(4): 1123S-1127S.

10. Buman MP, Yasova D, Giacobbi PR. Descriptive and narrative reports of barriers and motivators to physical activity in sedentary older adults. Psychol Sport Exerc 2010; 11:223-30.

11. Breen L, Phillips SM. Interactions between exercise and nutrition to prevent muscle waste during ageing. $\mathrm{Br} \mathrm{J}$ Clin Pharmacol. 2013;75(3):708-715.

12. Nascimento CM, Ingles M, et.al. Sarcopenia, frailty and their prevention by exercise. Free Radical Biology and Medicine. 2019; 132:42-49

13. Candow DG, Chilibeck PD, Abeysekara S, Zello GA. Short-term heavy resistance training eliminates age-related deficits in muscle mass and strength in healthy older males. J Strength Cond Res. 2011;25(2):326-333.

14. Hong Jeeyoung, Jeongeun Kim RN, Suk Wha Kim and Hyoun-Joong Kong. 2016. Effects of home-based teleexercise on sarcopenia among community dwelling elderly adults: Body composition and functional fitness. Experimental Gerontology 2016;11:002.

15. Tsekoura, Maria Evdokia Billis, Elias Tsepis, Zacharias Dimitriadis, Charalampos Matzaroglou, Minos Tyllianakis, Elias Panagiotopoulos and John Gliatis. The Effects of Group and Home-Based Exercise Programs in Elderly with Sarcopenia: A Randomized Controlled Trial. J. Clin. Med. 2018; (7): 480
16. Landi F, Calvani R, Picca A, Tosato M, Martone AM, D'Angelo E, et al. Impact of habitual physical activity and type of exercise on physical performance across ages in community-living people. PLoS ONE 2018; 13(1): e0191820.

17. Perkin, Oliver J. Rebecca L. Travers, Javier T. Gonzalez, James E. Turner, Fiona Gillison, Cassie Wilson, Polly M. McGuigan,Dylan Thompson1 and Keith A. Stokes. Exercise Strategies to Protect Against The Impact Of ShortTerm Reduced Physical Activity On Muscle Function And Markers Of Health In Older Men: Study Protocol For A Randomised Controlled Trial. Biomed Central 2016; 17:381

18. Maruya, Kohei, Yasuyoshi Kawa hideaki ishibashi, hiroaki Fujita tomoyukaria haruyasu and Maguchi. Effect of a simple and adherent home exercise program on the physical function of community dwelling adults sixty years of age and older with pre-sarcopenia or sarcopenia. J. Phys. Ther. Sci; 2016; (28): 3183-3188,

19. Yuki, Atsumu, Fujiko Ando Rei Otsuka and Hiroshi Shimokata. Sarcopenia based on the Asian Working Group for Sarcopenia criteria and all-cause mortality risk in older Japanese adults. Geriatr Gerontol Int 2017 ; 17(10) 16421647

20. Bull, Fiona C., Salih S Al-Ansari, Stuart Biddle, Katja Borodulin,Matthew P Buman, Greet Cardon,Catherine Carty,Jean-Philippe Chaput ,Sebastien Chastin ,Roger Chou, Paddy C Dempsey, Loretta DiPietro,Ulf Ekelund, Joseph Firth, Christine M Friedenreich, Leandro Garcia,Muthoni Gichu, Russell Jago Peter T Katzmarzyk, Estelle Lambert, Michael Leitzmann, Karen Milton Francisco B Ortega, Chathuranga Ranasinghe, Emmanuel Stamatakis, Anne Tiedemann, Richard P Troiano Juana F Willumsen, Hidde P van der Ploeg, Vicky Wari. World Health Organization 2020 Guidelines on physical activity and sedentary behavior. Br J Sports Med 2020: 54:14511462.

21. Eriksen CS, Garde E, Reislev NL, Cathrine Lawaetz Wimmelmann, Theresa Bieler,Andreas Kraag Ziegler, Anne Theil Gylling,Kasper Juel Dideriksen, Hartwig Roman Siebner,Erik Lykke Mortensen, Michael Kjaer. Physical activity as intervention for age-related loss of muscle mass and function: protocol for a randomised controlled trial). BMJ Open $2016 ; 6: \mathrm{e} 012951$.

22. Belza, Basia, RN, Julie Walwick, MSW, Sharyne ShiuThornton, , Sheryl Schwartz, Mary Taylor, James LoGerfo. Older Adult Perspectives on Physical Activity and Exercise: Voices from Multiple Cultures. Prev Chronic Dis [serial online] 2004; 1(4)

23. Miyazakia Ryo. Taro Takeshimab, Kazuhiko Kotani. Exercise Intervention for Anti-Sarcopenia in CommunityDwelling Older People. J Clin Med Res. 2016;8(12):848853.

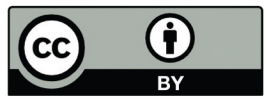

This work is licensed under a Creative Commons Attribution 